Portland State University

PDXScholar

3-1-1982

\title{
Comparison of Bethe's formula for magnetic- moment transitions in atoms with the generalized Rosenbluth's equation
}

\author{
P.T. Leung \\ Portland State University \\ M. L. Rustgi \\ J. E. Turner
}

Follow this and additional works at: https://pdxscholar.library.pdx.edu/phy_fac

Part of the Physics Commons

Let us know how access to this document benefits you.

\begin{abstract}
Citation Details
Leung, P. T., Rustgi, M. L., \& Turner, J. E. (1982). Comparison of Bethe's formula for magnetic-moment transitions in atoms with the generalized Rosenbluth's equation. Physical Review A (General Physics), 25(3), 1775-1776.
\end{abstract}

This Article is brought to you for free and open access. It has been accepted for inclusion in Physics Faculty Publications and Presentations by an authorized administrator of PDXScholar. Please contact us if we can make this document more accessible: pdxscholar@pdx.edu. 


\title{
Comparison of Bethe's formula for magnetic-moment transitions in atoms with the generalized Rosenbluth's equation
}

\author{
P. T. Leung and M. L. Rustgi \\ Physics Department, State University of New York at Buffalo, Amherst, New York 14260
}

J. E. Turner

Health and Safety Research Division, Oak Ridge National Laboratory, Oak Ridge, Tennessee 37830

(Received 6 October 1981)

\begin{abstract}
An error in an old but often quoted paper by Bethe on the cross section for an atomic transition caused by the magnetic moment of the projectile is corrected by comparing it with the generalized Rosenbluth's formula.
\end{abstract}

In an often quoted paper, ${ }^{1}$ Bethe $^{2}$ worked out the differential cross section for the scattering of a neutrino from atomic electrons via its magnetic moment and used it to set limits on the possible neutrino magnetic moment by employing the upper bounds for the ionization power in such a process. In 1950, prompted by the then new results in quantum electrodynamical radiative corrections, Rosenbluth $^{3}$ derived the elastic cross section for a free electron scattered from a free proton incorporating the effective charges and the anomalous magnetic moment of the proton. In this note, we shall compare Bethe's original result with an extended version of Rosenbluth's equation to electron transitions in atoms and conclude that there is an error in Bethe's result. The correct result is reported.

Bethe's original form for the differential cross section for atomic transition induced by an incident neutrino via its magnetic moment $\lambda$ reads [Eq. (2) of Ref. 2]

$$
\frac{d \sigma}{d \Omega}=\left(\left.\frac{e \lambda}{\hbar c}\right|^{2} \frac{\left|\overrightarrow{\mathbf{P}}^{\prime}\right|}{|\overrightarrow{\mathbf{P}}|} \frac{\left[\left(E+E^{\prime}\right)^{2}-4 \mu^{2} c^{4}\right] S_{w}^{2}-c^{2}\left(\overrightarrow{\mathbf{P}}+\overrightarrow{\mathbf{P}}^{\prime}, \overrightarrow{\mathbf{s}}_{w}\right)^{2}+4 \mu^{2} c^{4} \overrightarrow{\mathbf{s}}_{w}^{2}}{c^{2}\left(\overrightarrow{\mathbf{P}}-\overrightarrow{\mathbf{P}}^{\prime}\right)^{2}-\left(E-E^{\prime}\right)^{2}},\right.
$$

where $(E, \overrightarrow{\mathbf{P}})$ and $\left(E^{\prime}, \overrightarrow{\mathbf{P}}^{\prime}\right)$ are, respectively, the energy and momentum of the incident and the scattered neutrino and $\mu$ is the rest mass of the neutrino. $S_{w}$ and $\overrightarrow{\mathrm{s}}_{w}$ are the atomic matrix elements defined by

$$
\begin{aligned}
& S_{\dot{w}}=\int e^{i \overrightarrow{\mathrm{q}} \cdot \overrightarrow{\mathrm{r}} / h} \phi_{w}^{*} \phi_{0} d \tau, \\
& \overrightarrow{\mathrm{s}}_{w}=\int e^{i \overrightarrow{\mathrm{q}} \cdot \overrightarrow{\mathrm{r}} / h} \phi_{w}^{*} \vec{\alpha} \phi_{0} d \tau,
\end{aligned}
$$

where $\overrightarrow{\mathrm{q}}=\overrightarrow{\mathrm{p}}-\overrightarrow{\mathrm{p}}^{\prime}$ is the momentum transfer, $\vec{\alpha}$ the Dirac matrices, and $\phi_{0}$ and $\phi_{w}$ are, respectively, the initial- and final-state wave functions of the atomic electron. Following Bjorken and Drell's ${ }^{4}$ notation, setting $\hbar=c=1$, regrouping terms and using cgs unit for $e$, (1) may be rewritten as

$$
\frac{d \sigma}{d \Omega}=\left(\frac{K e^{2}}{2 M}\right)^{2} \frac{\left|\overrightarrow{\mathbf{P}}^{\prime}\right|}{|\overrightarrow{\mathbf{P}}|} \frac{\left\{\left(E+E^{\prime}\right)^{2} S_{w}^{2}-\left[\left(\overrightarrow{\mathbf{P}}+\overrightarrow{\mathbf{P}}^{\prime}\right) \cdot \overrightarrow{\mathbf{s}}_{w}\right]^{2}\right\}-4 M^{2}\left(S_{w}^{2}-\overrightarrow{\mathbf{s}}_{w}^{2}\right)}{-q^{2}},
$$

where $\lambda=K e / 2 M$, and $\left.q^{2} \equiv\left(E-E^{\prime}\right)^{2}-\overrightarrow{(\vec{P}}-\overrightarrow{\mathrm{P}}^{\prime}\right)^{2} \equiv Q^{2}-\overrightarrow{\mathrm{q}}^{2}<0$ is the four-momentum transfer. We have replaced $\mu$ by $M$. In the high- $Q$ limit, when the atomic electron can be replaced by Dirac plane waves and an average over the electron's initial state and sum over its final state is carried out, Bethe's formula gives

$$
\frac{d \sigma}{d Q}=4 \pi\left(\frac{K e^{2}}{2 M}\right)^{2} \frac{1}{Q} \frac{m}{m+Q} \frac{E(E-Q)-M^{2}}{E^{2}-M^{2}},
$$


where $m$ is the mass of the electron [Eq. (21) of Ref. 2]. The purpose of this note is to show that Eq. (1) is in error via its comparison with the generalized Rosenbluth formula. ${ }^{3}$

By writing the proton current as ${ }^{3,4}$

$$
\bar{u}^{\prime} \Gamma_{\mu} u=e \bar{u}^{\prime}\left(\gamma_{\mu} F_{1}+\frac{i \sigma_{\mu \nu} q^{v}}{2 M_{p}} K F_{2}\right) u,
$$

where $F_{1}$ and $F_{2}$ are the Dirac and Pauli form factors, respectively, Rosenbluth's formula for a free electron interacting with a free proton of mass $M_{p}$ with the proton initially at rest is given by ${ }^{3,4}$

$$
\frac{d \sigma}{d \Omega}=\frac{\alpha^{2}}{4 E^{2}}\left\{1 / \sin ^{4}\left(\frac{\theta}{2}\right)\left[1+\left(\frac{2 E}{M_{p}}\right) \sin ^{2} \frac{\theta}{2}\right]\right\}\left[\left(F_{1}^{2}-\frac{K^{2} q^{2}}{4 M_{p}^{2}} F_{2}^{2}\right) \cos ^{2} \frac{\theta}{2}-\frac{q^{2}}{2 M_{p}^{2}}\left(F_{1}+K F_{2}\right)^{2} \sin ^{2} \frac{\theta}{2}\right]
$$

For comparison with Bethe [Eq. (3)], Rosenbluth's formula has to be extended to the reference frame in which the proton is moving towards a bound electron. The result is

$$
\frac{d \sigma}{d \Omega}=\frac{2 e^{4}}{q^{4}} \frac{\left|\overrightarrow{\mathbf{P}}^{\prime}\right|}{|\overrightarrow{\mathbf{P}}|}\left[2(\underline{P} \cdot \underline{I})^{2}\left[F_{1}^{2}-\frac{K^{2} q^{2}}{4 M_{p}^{2}} F_{2}^{2}\right]+\frac{q^{2} I^{2}}{2}\left(F_{1}+K F_{2}\right)^{2}\right],
$$

where $\underline{P}=(E, \overrightarrow{\mathrm{P}}), I_{\mu} \equiv \int e^{i \overrightarrow{\mathrm{q}} \cdot \overrightarrow{\mathrm{r}}} \bar{\phi}_{f} \gamma_{\mu} \phi_{i} d \tau$ is a four vector that corresponds to Bethe's $S_{w}$ and $\overrightarrow{\mathrm{s}}_{w}$ in (2) for $\mu=0$ and $\mu=k(1,2,3)$, respectively. When the electron wave functions $\phi_{i}$ and $\phi_{f}$ are replaced by plane waves and a transformation is made back to the frame in which the proton is initially at rest, Eq. (7) reproduces Eq. (6).

In order to compare (7) with Eq. (1) which applies to a neutral point magnetic moment as the projectile, we have to set $M_{p}=M, F_{1}=0$, and $F_{2}=1$. Equation (7) then reduces to

$$
\frac{d \sigma}{d \Omega}=\left(\frac{K e^{2}}{2 M}\right)^{2} \frac{\left|\overrightarrow{\mathbf{P}}^{\prime}\right|}{|\overrightarrow{\mathbf{P}}|} \frac{\left[\left(\underline{P}+\underline{P}^{\prime}\right) \cdot \underline{I}\right]^{2}-4 M^{2} I^{2}}{-q^{2}},
$$

where we have made use of the conservation of current:

$$
\left(\underline{P}-\underline{P}^{\prime}\right) \cdot I=\underline{q} \cdot \underline{I}=\int e^{i \overrightarrow{\mathrm{q}} \cdot \overrightarrow{\mathrm{r}}} \bar{\Phi}_{f}\left(q^{\mu} \gamma_{\mu}\right) \phi_{i} d \tau=0
$$

to write $2(\underline{P} \cdot \underline{I})=\underline{P} \cdot \underline{I}+\underline{P}^{\prime} \cdot \underline{I}$. In terms of the $S_{w}$ and $\overrightarrow{\mathrm{s}}_{w}$ in (2), Eq. (8) may be written as

$$
\frac{d \sigma}{d \Omega}=\left(\frac{K e^{2}}{2 M}\right)^{2} \frac{\left|\overrightarrow{\mathbf{P}}^{\prime}\right|}{|\overrightarrow{\mathbf{P}}|} \frac{\left[\left(E+E^{\prime}\right) S_{w}-\left(\overrightarrow{\mathbf{P}}+\overrightarrow{\mathbf{P}}^{\prime}\right) \cdot \overrightarrow{\mathbf{s}}_{w}\right]^{2}-4 M^{2}\left(S_{w}^{2}-\overrightarrow{\mathbf{s}}_{w}^{2}\right)}{-q^{2}}
$$

Comparison of (3) and (10) shows that Bethe's original result should be corrected in the manner shown above in (10). This result is consistent with Eq. (5) in Ref. 5.

${ }^{1}$ See, for example, C. L. Cowan and F. Reines, Phys. Rev. 107, 528 (1957).

${ }^{2}$ H. A. Bethe, Proc. Cambridge Philos. Soc. $\underline{31}, 108$ (1935).

${ }^{3}$ M. N. Rosenbluth, Phys. Rev. 무, 615 (1950).
4J. D. Bjorken, and S. D. Drell, Relativistic Quantum Mechanics (McGraw-Hill, New York, 1964).

${ }^{5}$ M. L. Rustgi, P. T. Leung, J. E. Turner, and W. Brandt, Phys. Rev. A 24, 2425 (1981). 